\title{
Accelerating the De-Personalization of Medicine: The Ethical Toxicities of COVID-19
}

\author{
Mark Arnold (D) - Ian Kerridge
}

Received: 24 May 2020 / Accepted: 6 August 2020

(C) Journal of Bioethical Inquiry Pty Ltd. 2020

\begin{abstract}
The COVID-19 pandemic has, of necessity, demanded the rapid incorporation of virtual technologies which, suddenly, have superseded the physical medical encounter. These imperatives have been implemented in advance of evaluation, with unclear risks to patient care and the nature of medical practice that might be justifiable in the context of a pandemic but cannot be extrapolated as a new standard of care. Models of care fit for purpose in a pandemic should not be generalized to reconfigure medical care as virtual by default, and personal by exception at the conclusion of the emergency.
\end{abstract}

Keywords COVID-19 · Clinical ethics · Ontology · Epistemology

The COVID-19 pandemic has created unprecedented challenges - challenges that are not simply public health

\footnotetext{
M. Arnold $(\bowtie)$

School of Rural Health (Dubbo/Orange), Sydney Medical School, Faculty of Medicine and Health, University of Sydney, PO BOX 1043, Dubbo, NSW 2830, Australia

e-mail: mark.arnold@sydney.edu.au

M. Arnold

Sydney Health Ethics, Faculty of Medicine and Health, University of Sydney, Campertown, NSW 2006, Australia

I. Kerridge

Sydney Health Ethics, Faculty of Medicine and Health, Haematology Department, Royal North Shore Hospital, University of Sydney, Sydney, NSW, Australia
}

and clinical ones but ethical ones (Klopfenstein 2008; Uscher-Pines et al. 2007; Afolabi 2018).

Ethical issues surrounding the provision of healthcare in pandemics are, of course, not new. Issues surrounding decisions about emergency resource allocation, access to mechanical ventilation, advance care directives and palliative care, both for infected and noninfected patients, were highlighted by the severe acute respiratory syndrome coronavirus (SARS) and Ebola virus disease epidemics (Curtis, Kross, and Stapleton 2020). These infectious outbreaks also brought into sharper focus "new" ethical challenges relating to constraint on human movement and interactions on paternalistic or communitarian grounds (Kotalik 2005; Conly 2014; Scoccia 2013; DeMaria 2013; Glod 2008). But in the years since, these and other related issues, including the effects of pandemics on the health workforce as a consequence of repetitive moral distress (Biddison et al. 2014; Whitehead et al. 2015; Lamiani, Borghi, and Argentero 2017), erosion of empathy (Decety and Cowell 2014), and physician burnout (Dzeng and Curtis 2018), largely faded from public view. COVID19 has, more starkly than at any time in the past century, brought these issues into sharp focus.

The ethics of triage and resource allocation have dominated public and professional discourse since the COVID-19 pandemic began and well before (Biddison et al. 2014). However, triage and resource allocation are not the sole ethical challenges that clinicians and health systems will face during the pandemic and into the future. In this paper we discuss the ethical consequences of changes to the way that healthcare is delivered and 
experienced in response to the threat of COVID-19. We suggest that models of care that are justifiable in the context of a pandemic should not, by default, become the new standard of care when the pandemic has passed. While COVID-19 will undoubtedly raise questions about standard practice and promote consideration of entirely different processes and policies for healthcare delivery, many of the strategies put in place in the pandemic remain inadequately assessed and validated. Many create both well-recognized and previously unforeseen harms resulting from quarantine and isolation, disengagement with the corporeal patient, the privileging of data, and the incorporation of unproven physical and virtual technologies.

\section{The Effects of Quarantine and Isolation}

While there are clear public health justifications to support quarantine during pandemics and infectious outbreaks, quarantine may also have myriad adverse effects on those who are quarantined, including heightened uncertainty, anxiety, depression, isolation, frustration, fear and stigmatization, concerns about privacy breaches and even post-traumatic stress disorder (Tracy, Rea, and Upshur 2009). It is well documented that public risk discourse (Lupton 1993) and the "dispassionate expertise" of public health practice may unintentionally marginalize, stigmatize, blame and exclude persons or groups (Lupton 2013, 1995), leading to demonization of certain groups at the beginning of the current pandemic (Lin 2020; Escobar 2020).

Importantly, isolation of patients who are perceived to present a risk to other patients, healthcare professionals, and/or to the wider community may also create a range of unintended harms. It has been extensively documented that isolated patients suffer a greater number of potentially preventable adverse events including medication errors, falls, sub-optimal documentation of care, and greater treatment dissatisfaction than nonisolated patients, along with stigmatization (Bryan, Call, and Elliott 2007). In philosophical terms, this suggests that while the precautionary principle as relevant to public health (which entails decisive preventive action in the context of uncertainty related to the extent and depth of risk) may justify isolation, resultant malign effects may be disingenuously claimed to be permissible through the doctrine of double effect (whereby morally undesirable harms that are foreseen but are not intended may be deemed morally acceptable).

\section{Changing Patterns of Hospital Care}

COVID-19 has radically transformed the way that healthcare is delivered. While hospitals have changed significantly since their emergence, what has characterized the change in clinical practice since COVID-19 has been the speed of change and the willingness to change without recognition of the harms that may result from the adoption of technologies and practices that are either unproven or known to have adverse consequences in other settings.

Increasing Utilization of Electronic Medical Records

Electonric Medical Records (EMRs) are increasingly a feature of the contemporary health landscape and characterize patient management in the context of COVID19-in large measure because EMR utilization reduces the risk of exposure via fomites between health workers, patients, and different parts of the healthcare service. But while this makes intuitive sense, a large body of literature on the impact of digitization, computerization, and EMR utilization on patient care makes clear that it has harms as well as benefits and may serve to reduce the quality of patient care. In this regard it is noteworthy that, increasingly, the first encounter with a newly admitted patient is electronic - an encounter with the 'iPatient' - the virtual construct of the patient in the computer (Verghese 2008), reflecting a process of “digitised embodiment" (Lupton 2014). Virtual patient rounding (Verghese 2008), associated minimalist physical interactions (Verghese et al. 2015), heavy reliance on EMR data which may not "capture and represent what clinicians are thinking about the patients and their problems" (Colicchio and Cimino 2018, 182), and "chart biopsy" (Hilligoss and Zheng 2012; Kendall et al. 2013) are new forms of behaviour facilitated by heavy reliance on the EMR.

Though it could be argued that implicit bias related to gender and minority groups might be lessened in virtual encounters, video-enabled interactions will still give indicators of sex, age, ethnicity and other sources of potential bias to participants. Inferences regarding age, sex, and ethnicity may also be conveyed through verbal clues relating to voice pitch, inflection, grammar, 
accent, and so forth. These matters have not been systematically addressed. What is unique to virtual interactions is that acontextual data interpretation-occurring in the absence of the physical patient - introduces decisional and attributional cognitive biases (Croskerry 2003) with the potential for tangible patient harms. For instance, the "cloning" of EMR history and clinical examination findings from prior records is well recognized (Thielke, Hammond, and Helbig 2007). It may be a source of compounding errors (Rethans, Martin, and Metsemakers 1994) and medicolegal incidents (Pesto, Mittenfelner, and Spaedy 2018) and may create a powerful disincentive to the accrual and incorporation of freshly derived data about a patient's illness and management (Thielke, Hammond, and Helbig 2007; Bowman 2013). More fundamentally, acontextual management-without physical reference to the patient - also may erode important ethical, ontological, and epistemological dimensions of the clinical encounter, which gains its strength from the act of interpretation "which draws on narrative skills to integrate the overlapping stories told by patients, clinicians, and test results" (Greenhalgh 1999, "Summary Points").

\section{Foregoing Physical Examination}

Patients who are isolated during a pandemic, by definition, have less physical contact with others. Somewhat paradoxically, this may also include less contact with health practitioners who record clinical data, utilize an array of investigations to guide their management, and observe patients without actually touching them. This may mean that patients are also less often examined. Again, while this may make intuitive sense and be a justifiable response to the need to contain a pathogen, inadequate or absent physical examination has been clearly identified as a source of patient harm by increasing diagnostic errors, reducing efficiency, compromising the quality of clinical decision-making (Verghese et al. 2015), and making medical care akin to "palm reading, observation, and intuition" or medical fortune-telling (Alpert 2015). Physical disengagement from patients may also have other more profound effects on patient care and the experience of care as the disembodiment, "datafication" (Fortin 2015), and "data fetishisation" that accompanies it (Storni 2015). The de-facto substitution of patient "charts" as surrogates for patients, also serves to exclude narrative and the personalization of care, diminishing and even "destroy(ing) ... every kind of subjectivity" (Gremy, Fessler, and Bonnin 1999) and opportunities to develop interpersonal trust and empathy (Lee and Alkureishi 2017).

It should also be appreciated that a lack of appropriate personal protective equipment (PPE) for healthcare professionals and other essential workers will necessitate practitioners limiting their exposure to patients to reduce their personal risk of infection. Virtual interactions do not diminish the requirement of systems to provide workers with necessary PPE. Normative depersonalization will arise through necessity.

Interposing Technology: The use of Workstations-on-Wheels (WOWs) or Computers-on-Wheels (COWs)

Computers-on-Wheels or Workstations-on-Wheels (hereafter COWS) are mobile nodes of a hospital's electronic medical record (EMR)/patient management system that are wheeled by medical teams from one person's bedside to another during ward rounds. As vehicles of the EMR, they have largely replaced the written medical record transported by staff. They are ubiquitous in contemporary healthcare settings and are a cornerstone of the clinical management of patient during pandemics. But notwithstanding their evident utility as mobile data repositories, COWs have potentially negative effects on clinical care and, ironically, may act as fomites, particularly when insufficiently decontaminated (Codish et al. 2015; Beam et al. 2016) and used by multiple persons (Po et al. 2009; Tan et al. 2013; Pyrek 2014). Importantly, experience with COWS in non-pandemic settings makes clear that, like many other technological interventions in healthcare, they may not provide the benefits promised or, at the very least, may also have deleterious effects on the design, delivery, and experience of healthcare. Indeed, studies of COWS suggest that they may be poorly manoeuvrable, un-ergonomic, and variably fit for purpose (Debono et al. 2017), create duplicative work (Tang and Carpendale 2008), become an additional stressor in an already stressful work environment (Öberg et al. 2018), and create an unnecessary distraction from patient care (Bertman 2017; Dhillon et al. 2018; Dhillon, Gewertz, and Ley 2019). More importantly, they may create barriers to patient care, promote de-skilling of health professionals, lesson contact time with patients, reduce communication with patients and families and entrench conventional role demarcation and status within 
healthcare teams, thereby reducing the quality of care, reinforcing the hidden curriculum, and eroding the critical learning of all health professionals (Manias et al. 2020).

\section{Telehealth as the New Norm of Care in the Wake of COVID-19}

While telehealth has been incorporated into systems of healthcare the world over, particularly in countries with large rural and remote populations and where healthcare must be delivered over large distances, the COVID-19 pandemic has greatly accelerated the adoption of telehealth as a new standard of care over a matter of weeks. Literally overnight (Mehrotra et al. 2020), and without careful evaluation of its benefits and risks, the medical profession has adopted telehealth in preference to face-to-face clinical encounters to protect themselves and their patients. In the current Australian context, telephone calls may be construed as "telehealth" which does not represent the generally accepted interaction referred to in the literature ("optimal telehealth").

In this regard it is noteworthy that a number of health systems analysts, including Hollander and Carr (2020) have commented on the potential role for telemedicine and artificial intelligence/machine learning to contribute to healthcare systems' responses to COVID-19. Indeed, for this purpose, it may be ideally suited as it can be rapidly deployed, requires only mobile phone or computer access, is ultimately portable, enables remote supervision of non-critically ill patients, and, when combined with easily sterilizable tablet computers, allows telehealth visits even for patients in isolation. Further, monitoring programmes have been established for intensive care units permitting medical and nursing staff to remotely monitor multiple patients across multiple sites (Hollander and Carr 2020), despite the fact that for persons requiring critical care interventions, human-tohuman contact with appropriate infection precautions is unavoidable.

The authors conclude that: "Disasters and pandemics pose unique challenges to health care delivery. Though telehealth will not solve them all, it's well suited for scenarios in which infrastructure remains intact and clinicians are available to see patients" (Hollander and Carr 2020, 1681).

There are, however, other aspects of telehealth that make it suitable not just for healthcare delivery during a pandemic but also for neoliberal appropriation, since telehealth may be (relatively) inexpensive, may extend the reach of government and healthcare providers into hitherto private spaces, may provide a means for controlling and limiting the healthcare workforce, and may privilege health services over care, communication, and interpersonal interaction.

Achieving cost savings through telehealth is, of course, not a negative thing as there are profound social benefits that accrue from reducing the costs of delivering specialized care, particularly remotely, and from reducing the direct and indirect costs experienced by patients, their families and communities.

Given the desire of governments of all political persuasions to limit health expenditure and the enormous financial impact of COVID-19, it seems likely that in the (near) future, cost containment imperatives may be leveraged to control workforce numbers and their distribution (Garson Jr 2013) and to manage and limit the cost of healthcare. Again, while telehealth may provide a means by which the costs of healthcare are reducedincluding the cost of pre-hospital emergency medical services (Langabeer et al. 2017), post-operative care (Nandra et al. 2019), and community care (Jacobs et al. 2019) during and beyond the pandemic - the question remains as to whether this should be done and whether it can be done without sacrificing quality and integrity.

Though there is an emerging literature regarding the corrosive effect of telemedicine on empathy (Cain and Romanelli 2009; Terry and Cain 2016) there is relatively little examination of the longer-term effect that telemedicine may have on the quality of clinical interactions, on health-professional-patient relationships, on inter-professional care, and moral imagination, and on the myriad of ethical issues that surround and inscribe healthcare (Irvine 2005). While case conferences and other interdisciplinary interactions with patients can happen by telehealth, the typical telehealth consultation-however convenient for practitioner and patient - inevitably raises concerns relating to the quality of clinical care and decision-making, the quality of inter-professional interaction privacy, confidentiality, the potential loss of relevant information particularly through the unavailability of "electronically mediated interactions for physical examination," and disruption of the patient-physician dyad (Chaet et al. 2017). These concerns are relevant regardless of whether one is undertaking care in a pandemic or in situations of normality, and pertain to all persons, and are particularly stark 
"when someone is ill or otherwise vulnerable" (Kaplan and Litewka 2008).

Optimal telehealth requires optimal hardware, infrastructure, stable connectivity of sufficient bandwidth, training of providers, and facilitation usually by another healthcare professional at the patient end of the encounter. It is not possible to guarantee that the quality of the interaction is not compromised by implementation issues. In the example of rural Australia, these conditions are inconsistently deliverable (Gagnon et al. 2006). The implementation of telehealth may be optimal in facilities and systems which are highly resourced and can afford the associated costs to implement optimal telehealth with the aim of increasing efficiency and reducing care expenditure (Dorsey and Topol 2016). However, these means of funding telehealth implementation are not universally available. Furthermore, entrepreneurial models of telehealth provision threatens - through its apparent availability and low cost - to exploit "price-sensitive" and vulnerable patients. These people may be tempted by price signals to disengage from established care networks leading to over-servicing and potentially receive shallow, transactional, fragmented, inappropriate, and ultimately dis-integrated care.

Despite the potential for extensive utilization of telehealth to adversely affect many aspects of healthcare, it already seems unlikely that the shift to telemedicine that has occurred since COVID-19 will be quickly reversed - even if there is evidence of disbenefit. In part this will be because of the costs savings resulting from telemedicine, but it may also occur because of a reluctance by third-party payers, healthcare providers, and even patients to return to "outmoded" and "inefficient" models of care. There are a number of reasons why patients may favour the implementation of more broadly available telehealth presently and after the conclusion of the pandemic. These include but are not limited to convenience, a reduction in opportunity costs related to travel to appointments, time off work, and care arrangements for family. Third party payers and health systems however generally do not factor these costs into account, but they are potent sources of patient dissatisfaction with the current model of face-to-face care delivery. In this context it is of concern that many patients qua healthcare consumers are unclear that there is no established correlation between being satisfied in regard to their care measured by satisfaction metrics and what is demonstrably high quality care (Arnold, Kerridge, and Lipworth 2020). What this means for healthcare providers (particularly those who work in nonnationalized fee-for-service health systems) and ultimately for patients remains to be seen.

\section{Conclusion}

The COVID-19 pandemic has demanded practical solutions to reduce the risk of transmission of infection to patients and healthcare professionals in both hospital and community settings. Within weeks, medical technologies and models of care which had been evolving, often with inconsistent and incomplete validation, were universally implemented. The effects of the pandemic on the health system and the experience of healthcare will remain long after its virological nadir, and it is distinctly possible that the model of medical care adopted in response to COVID-19 will be maintained as the new norm of care. It is important to consider whether the challenges to sustain healthcare systems and patient care arising from the effects of COVID-19 may be driven by technological imperatives and neoliberal governmentality. It remains for clinicians, researchers, and consumers to scrutinize whether this process meets with the ethical, clinical, and humanitarian concerns of medicine after the imperatives of simple survival have lapsed.

Acknowledgement The authors with to thank the two anonymous reviewers for their constructive criticism.

\section{References}

Afolabi, M.C. 2018. Pandemic influenza: A comparative ethical approach. In Public health disasters: A global ethical framework, 59-96. Advancing Global Bioethics (12). Cham: Springer International Publishing.

Alpert, J.S. 2015. Palm reading, observation, and intuition. The American Journal of Medicine 128(12): 1263-1264.

Arnold, M. H., I. Kerridge and W. Lipworth. 2020. An ethical critique of person-centred care. European Journal for Person Centered Healthcare (forthcoming).

Beam, E. L, S.G. Gibbs, A.L. Hewlett, et al. 2016. Evaluating isolation behaviors by nurses using mobile computer workstations at the bedside. CIN: Computers, Informatics, Nursing 34(9): 387-392.

Bertman, S. 2017. Electronic challenges to patient safety and care. In Distracted doctoring: Returning to patient-centered care in the digital age. Edited by P. J. Papadakos and S. Bertman, 17-28. Springer International Publishing. 
Biddison, L.D., K.A. Berkowitz, B. Courtney, et al. 2014. Ethical considerations: Care of the critically ill and injured during pandemics and disasters: CHEST Consensus Statement. Chest 146(4, Supplement): e145S-e155S.

Bowman, S. 2013. Impact of electronic health record systems on information integrity: Quality and safety implications. Perspectives in Health Information Management 10(Fall): $1 \mathrm{c.}$

Bryan, C.S., T.J. Call, and K.C. Elliott. 2007. The ethics of infection control: Philosophical frameworks. Infection Control \& Hospital Epidemiology 28(9): 1077-1084.

Cain, J., and F. Romanelli. 2009. E-professionalism: A new paradigm for a digital age. Currents in Pharmacy Teaching and Learning 1(2): 66-70.

Chaet, D., R. Clearfield, J.E. Sabin, and K. Skimming. 2017. Ethical practice in telehealth and telemedicine. Journal of General Internal Medicine 32(10): 1136-1140.

Codish, S., R. Toledano, V. Novack, et al. 2015. Effectiveness of stringent decontamination of computer input devices in the era of electronic medical records and bedside computing: A randomized controlled trial. American Journal of Infection Control 43(6): 644-646.

Colicchio, T.K, and J.J. Cimino. 2018. Clinicians' reasoning as reflected in electronic clinical note-entry and reading/retrieval: A systematic review and qualitative synthesis. Journal of the American Medical Informatics Association 26(2): 172184.

Conly, S. 2014. Against autonomy: Justifying coercive paternalism. Journal of Medical Ethics 40(5): 349-349.

Croskerry, P. 2003. The importance of cognitive errors in diagnosis and strategies to minimize them. Academic Medicine 78(8): 775-780.

Curtis, J.R., E.K. Kross, and R.D. Stapleton. 2020. The importance of addressing advance care planning and decisions about Do-Not-Resuscitate orders during novel coronavirus 2019 (COVID-19). JAMA 323(18): 1771-1772.

Debono, D., N. Taylor, W. Lipworth, et al. 2017. Applying the theoretical domains framework to identify barriers and targeted interventions to enhance nurses' use of electronic medication management systems in two Australian hospitals. Implementation Science 12(1): 42-54.

Decety, J., and J.M. Cowell. 2014. The complex relation between morality and empathy. Trends in Cognitive Sciences 18(7): 337-339.

DeMaria, A.N. 2013. The nanny state and "coercive paternalism." Journal of the American College of Cardiology 61(20): 2108-2109.

Dhillon, N.K., S.E. Francis, J.M. Tatum, et al. 2018. Adverse effects of computers during bedside rounds in a critical care unit. JAMA Surgery 153(11): 1052-1053.

Dhillon, N.K., B.L. Gewertz, and E.J. Ley. 2019. Leveraging the right technology in health care-Having a Cow-Reply. JAMA Surgery 154(4): 365-366.

Dorsey, E.R., and E. J. Topol. 2016. State of telehealth. New England Journal of Medicine 375(2): 154-161.

Dzeng, E., and J.R. Curtis. 2018. Understanding ethical climate, moral distress, and burnout: A novel tool and a conceptual framework. BMJ Quality \& Safety 27: 766-770.

Escobar, N. 2020. When xenophobia spreads like a virus. Code Switch, March 4. National Public Radio. https://www.npr. org/2020/03/02/811363404/when-xenophobia-spreads-likea-virus. .

Fortin, S. 2015. Between reason, science and culture: Biomedical decision-making. Journal International de Bioethique et D'Ethique Des Sciences 26(4): 39-56.

Gagnon, M-P., J. Duplantie, J-P. Fortin, and R. Landry. 2006. Implementing telehealth to support medical practice in rural/ remote regions: what are the conditions for success? Implementation Science 1(1): 18.

Garson Jr, A.. 2013. New systems of care can leverage the health care workforce: How many doctors do we really need? Academic Medicine 88(12): 1817-1821.

Glod, W. 2008. How not to argue against paternalism. Reason Papers 30: 7-22.

Greenhalgh, T. 1999. Narrative based medicine in an evidence based world. BMJ 318(7179): 323-325.

Gremy, F., J.M. Fessler, and M. Bonnin. 1999. Information systems evaluation and subjectivity. International Journal of Medical Informatics 56(1-3): 13-23.

Hilligoss, B., and K. Zheng. 2012. Chart biopsy: An emerging medical practice enabled by electronic health records and its impacts on emergency department-inpatient admission handoffs. Journal of the American Medical Informatics Association 20(2): 260-267.

Hollander, J.E., and B.G. Carr. 2020. Virtually perfect? Telemedicine for COVID-19. New England Journal of Medicine 382: 1679-1681.

Irvine, R. 2005. Mediating telemedicine: Ethics at a distance. Internal Medicine Journal 35(1): 56-58.

Jacobs, J.C., J. Hu, C. Slightam, A. Gregory, and D.M. Zulman. 2019. Virtual savings: Patient-reported time and money savings from a VA national telehealth tablet initiative. Telemedicine and e-Health. ePub ahead of print, December 26. doi.org/10.1089/tmj.2019.0179.

Kaplan, B., and S. Litewka. 2008. Ethical challenges of telemedicine and telehealth. Cambridge Quarterly of Healthcare Ethics 17(4): 401-416.

Kendall, L., P. Klasnja, J. Iwasaki, et al. 2013. Use of simulated physician handoffs to study cross-cover chart biopsy in the electronic medical record American Medical Informatics Association (AMIA)Annual Symposium Proceedings: AMIA Symposium 2013: 766.

Klopfenstein, M.L. 2008. Pandemic influenza and the duty to treat: The importance of solidarity and loyalty. The American Journal of Bioethics 8(8): 41-43.

Kotalik, J. 2005. Preparing for an influenza pandemic: Ethical issues. Bioethics 19(4): 422-431.

Lamiani, G., L. Borghi, and P. Argentero. 2017. When healthcare professionals cannot do the right thing: A systematic review of moral distress and its correlates. Journal of Health Psychology 22(1): 51-67.

Langabeer, J.R., T. Champagne-Langabeer, D. Alqusairi, et al. 2017. Cost-benefit analysis of telehealth in pre-hospital care. Journal of Telemedicine and Telecare 23(8): 747-751.

Lee, W.W.. and M.L. Alkureishi. 2017. The impact of EMRs on communication within the doctor-patient relationship. In Distracted doctoring: Returning to patient-centered care in the digital age. Edited by P.J. Papadakos and S. Bertman, 101-120. Springer Internatonal Publishing.

Lin, C-Y. 2020. Social reaction toward the 2019 novel coronavirus (COVID-19). Social Health and Behavior 3(1): 1-2. 
Lupton, D. 1993. Risk as moral danger: The social and political functions of risk discourse in public health. International Journal of Health Services 23(3): 425-435.

. 1995. The imperative of health: Public health and the regulated body. London: Sage. .2013. Risk and emotion: Towards an alternative theoretical perspective. Health, Risk \& Society 15(8): 634-647. . 2014. Critical perspectives on digital health technologies. Sociology Compass 8(12): 1344-1359.

Manias, E., T. Bucknall, N. Wickramasinghe, K. Gray, J. Schaffer, and E. Rosenfeld. 2020. Patient and family engagement in communicating with electronic medical records in hospitals: A systematic review. International Journal of Medical Informatics 134: 104036.

Mehrotra, A., K. Ray, D. M. Brockmeyer, M.L. Barnett, and J.A. Bender. 2020. Rapidly converting to virtual practices: Outpatient care in the era of COVID-19. New England Journal of Medicine Catalyst Innovations in Care Delivery, April 1. doi: doi:https://doi.org/10.1056/CAT.20.0091.

Nandra, K., G. Koenig, A. DelMastro, E. A. Mishler, J. E. Hollander, and C. J. Yeo. 2019. Telehealth provides a comprehensive approach to the surgical patient. The American Journal of Surgery 218(3): 476-479.

Öberg, U., C.J. Orre, U. Isaksson, R. Schimmer, H. Larsson, and Å. Hörnsten. 2018. Swedish primary healthcare nurses' perceptions of using digital eHealth services in support of patient self-management. Scandinavian Journal of Caring Sciences 32(2): 961-970.

Pesto, M.M., J.I. Mittenfelner, and A. Spaedy. 2018. Legal concerns in complicated obstetrical cases. In The diagnosis and management of the acute abdomen in pregnancy, edited by P. B. Greenspan, 241-267. Cham: Springer International Publishing.

Po, J.L., R. Burke, C. Sulis, and P.C. Carling. 2009. Dangerous cows: An analysis of disinfection cleaning of computer keyboards on wheels. American Journal of Infection Control 37(9): 778-780.

Pyrek, K.M. 2014. Cross-contamination prevention: Addressing keyboards as fomites. Infection Control Today: Special Report (May). https://www.cleanside.fi/wpcontent/uploads/2016/02/ICT_Cross-contaminationkeyboards.pdf. .

Rethans, J-J., E. Martin, and J. Metsemakers. 1994. To what extent do clinical notes by general practitioners reflect actual medical performance? A study using simulated patients. British Journal of General Practice 44(381): 153-156.

Scoccia, D. 2013. The right to autonomy and the justification of hard paternalism. In Paternalism: Theory and practice, edited by C. Coons and M. Weber, 74-92. Cambridge University Press.

Storni, C. 2015. Patients' lay expertise in chronic self-care: A case study in type 1 diabetes. Health Expectations 18(5): 14391450 .

Tan, T.Y., J.S.M. Tan, H. Tay, G.H. Chua, L.S.Y. Ng, and N. Syahidah. 2013. Multidrug-resistant organisms in a routine ward environment: differential propensity for environmental dissemination and implications for infection control. Journal of Medical Microbiology 62(5): 766-772.

Tang, C., and S. Carpendale. 2008. Evaluating the deployment of a mobile technology in a hospital ward. CSCW '08: Proceedings of the 2008 ACM conference on Computer supported cooperative work: 205-214. New York: Association for Computing Machinery. https://doi. org/10.1145/1460563.1460596.

Terry, C., and J. Cain. 2016. The emerging issue of digital empathy. American Journal of Pharmaceutical Education 80(4): 58 .

Thielke, S., K. Hammond, and S. Helbig. 2007. Copying and pasting of examinations within the electronic medical record. International Journal of Medical Informatics 76: S122-S128.

Tracy, C.S., E. Rea, and R.E.G. Upshur. 2009. Public perceptions of quarantine: Community-based telephone survey following an infectious disease outbreak. BMC Public Health 9(1): 470-477.

Uscher-Pines, L., P.S. Duggan, J.P. Garron, R.A. Karron, and R.R. Faden. 2007. Planning for an influenza pandemic: Social justice and disadvantaged groups. The Hastings Center Report 37(4): 32-39.

Verghese, A. 2008. Culture shock-Patient as icon, icon as patient. New England Journal of Medicine 359(26): 2748-2751.

Verghese, A., B. Charlton, J.P. Kassirer, M. Ramsey, and J.P.A. Ioannidis. 2015. Inadequacies of physical examination as a cause of medical errors and adverse events: A collection of vignettes. The American Journal of Medicine 128(12): 13221324.

Whitehead, P.B., R.K. Herbertson, A.B. Hamric, E.G. Epstein, and J.M. Fisher. 2015. Moral distress among healthcare professionals: Report of an institution-wide survey. Journal of Nursing Scholarship 47(2): 117-125.

Publisher's note Springer Nature remains neutral with regard to jurisdictional claims in published maps and institutional affiliations. 